\title{
2. Theoretisch-methodischer Einstieg
}

[...] any sustained account of the world is dense with storytelling. >Reality is not compromised by the pervasiveness of narrative; one gives up nothing except the illusion of epistemological transcendence, by attending closely to the stories.

Donna J. Haraway (1997: 64)

Donna J. Haraway hat sich in ihren Arbeiten für eine Abkehr von einem reflexiven Paradigma in der wissenschaftlichen Praxis zugunsten einer diffraktiven Denkweise ausgesprochen. In ihrer Kritik am reflexiven Paradigma schärft sie den Blick dafür, dass es sich bei wissenschaftlichen Perspektiven vor allem um Lese- und Schreibtechnologien handelt, denen Verfahren und Methoden zugrunde liegen, die ein bestimmtes Wissen und daraus hervorgehend bestimmte Sichtweisen und Realitäten evozieren. Sie argumentiert für die Vorstellung umstrittener narrativer Felder von Bedeutungen in der Konstruktion wissenschaftlichen Wissens (vgl. Haraway 1989: 255). Dabei sind >stories` und >storytelling durchgängige Motive ihrer Arbeiten. So spricht sie in ihren frühen Schriften bspw. von den »narratives of scientific fact (ebd.: 5), von »story-telling practices (ebd.) und dem wissenschaftlichen Diskurs als "story telling (ebd.: 6). Derselbe Leitgedanke findet sich auch in ihrer aktuellen Arbeit, wenn es bspw. heißt: »It matters which stories tell stories, which concepts think concepts. (Haraway 2016: 101)

Mit dieser Vorstellung des Geschichtenerzählens als Wissenspraktik eröffnet sich die Möglichkeit die diskursive und ästhetische Dimension der Bedeutungsund Wissensgenerierung zusammen $\mathrm{zu}$ denken und $\mathrm{zu}$ analysieren. In Haraways Betrachtung sind hierfür zwei Vorstellungen bzw. Konzepte zentral. Zum einen das Konzept der Figurationen bzw. Figuren, die sie als performative, verbale wie auch visuelle Bilder entwirft, in denen sich die materiell-semiotischen Praktiken der Bedeutungs- und Wissensgenerierung verdichten. Zum anderen greift sie auf das bereits erwähnte Bild der Diffraktion zurück, das zu einem übergreifenden Motiv ihrer perspektivischen Ausrichtung avanciert. Haraways Antrieb für die Verfolgung einer diffraktiven Perspektive liegt nicht zuletzt darin, andere Sichtweisen 
und Realitäten durch die wissenschaftliche Betrachtung und Beschreibung hervorzubringen und eben nicht in eine Reproduktion des Immergleichen - des eigenen Spiegelbilds - zu verfallen. Denn hier sieht sie die Gefahr, dass eine kritische Praxis, die die Verbindung mit Anderen/m sucht, durch die Sorge um Kopie und Original und die Suche nach dem Authentischen und dem wirklich Wirklichen verstellt wird (vgl. Haraway 1997: 16 u. 273). Haraway verortet sich damit in einer feministischen Tradition, die eine selbstkritische Praxis der Differenz und Konnektivität verfolgt; eines Ich und Wir, das niemals mit sich selbst identisch ist und darauf hoffen kann, Verbindungen mit Anderen/m einzugehen (vgl. Haraway 2004 [1992]: 48). Mit ihrer diffraktiven Perspektive schlägt Haraway - kurz gesagt - eine wissenschaftliche Praxis »for doing [...] knowledge differently« (Schneider 2002: 466) vor.

Vor diesem Hintergrund soll Haraways Ansatz als Ausgangspunkt für den Entwurf einer diffraktiven Forschungsperspektive genutzt werden, die in der Lage ist, das Phänomen der Handy-Todesvideos in seiner Situiertheit und Komplexität an Videoereignissen und Bedeutungen sowie seiner Verbundenheit mit einer Ästhetisierung und Politisierung des Todes zu betrachten und beschreiben. Im Folgenden wird zunächst Haraways diffraktive Perspektive gemeinsam mit ihrer narrativen Konzeption der Figuration vorgestellt, die sie prominent in ihrer Arbeit Modest_Witness@Second_Millenium.FemaleMan@_Meets_Onco-Mouse ${ }^{\text {TM }}$ (1997) einsetzt. Insbesondere die Figuration bzw. Figur prägt seither ihre Auseinandersetzungen mit den Science-Fiction-Geschichten der Wissenschaft (vgl. hierzu auch Haraway 2016: 101). In einem zweiten Schritt wird Haraways Verständnis des situierten Wissens und der Kontingenz des Wissens fokussiert, das zentral in der perspektivischen Ausrichtung dieser Arbeit als einen diffraktiven Zugang zu einer figurativ-narrativen Praxis der Wissens- und Bedeutungsgenerierung ist. In den letzten beiden Abschnitten dieses Kapitels wird der konkrete Entwurf einer solchen diffraktiven Forschungsperspektive in Bezug auf das Phänomen der HandyTodesvideos und dessen methodische Umsetzung in dieser Arbeit vorgestellt.

\subsection{Diffraktion statt Reflexion: Geschichten, Figurationen und Bedeutungen}

In ihrer Argumentation setzt Haraway (1992 \& 1997) das optische Bild der Diffraktion dem der Reflektion entgegen. Diffraktion beschreibt in der Physik das Verhalten von Wellen in einem Medium wie Wasser oder Luft, wenn diese auf ein Hindernis treffen und gebeugt werden, d.h. wenn sie von ihrer ursprünglichen Bewegung abweichen. Treffen bspw. Meereswellen auf Felsen breiten sich kugelförmige Wellen im Wasser aus, die ein kreisförmiges Muster mit den Felsen als Mittelpunkt auf die Wasseroberfläche zeichnen. Durch die Beugung erreichen die Wellen Berei- 
che, von denen sie ursprünglich abgeschirmt waren. Das gleiche Phänomen lässt sich beobachten, wenn Regentropfen auf eine Wasseroberfläche auftreffen: Mit jedem Tropfen entstehen kreisförmige Wellen auf der Oberfläche, die die Einwirkung der Ereignisse als sich raumzeitlich ausbreitende Veränderungen in Form einer Berg- und Tallandschaft nachzeichnen. Mit zunehmender Entfernung vom Einwirkungspunkt werden die Wellenabstände größer und die Wellenberge kleiner bis die Höhen und Tiefen zu einer Ebene auslaufen und das Wasser zu seinem Ruhezustand zurückkehrt. Treffen zwei Wellen aufeinander interferieren sie konstruktiv oder destruktiv miteinander, d.h. die Wellen überlagern sich und verstärken oder schwächen sich gegenseitig: sie schlagen gemeinsam größere Wellen oder verstummen zu stillem Wasser. (vgl. Deuber-Mankowsky 2011: 89ff.; Spektrum 1998; Tipler 1994)

Wenn Wellen interferieren wirken sie also ineinander, indem sie zu etwas anderem werden und Differenzeffekte erzeugen. Anstatt beim Auftreffen auf ein Hindernis oder eine andere Welle in einem Vorgang der Reflektion auf sich selbst zurückgeworfen zu werden, verändern sie sich und gehen eine Art der Beziehung ein, die eine Vorstellung von Offenheit und relationalem Werden voraussetzt (vgl. Bath et al. 2013: 7). Relationalität hängt im Haraway'schen Denken »nicht von der Rekonstruktion des Ganzen ab, sondern von einer partialen Unabgeschlossenheit: Unabgeschlossen sein heißt relational, verbunden zu sein. (Trinkaus 2013:141) Unabgeschlossenheit ist für Haraway (1995: 231) überhaupt erst die Voraussetzung dafür, Relationen mit Anderen/m eingehen zu können. Diffraktion und Interferenz ${ }^{1}$ handeln damit nicht von einer Ausschlusslogik, sondern gehen von der Vorläufigkeit und Relationalität jedweder Grenzziehung aus, die einen Ausschluss selbst unmöglich, aber gerade dadurch die Politiken der Ausschließung analysierbar machen (vgl. Bath et al. 2013: 21).

Das im Begriff der Diffraktion enthaltene Bild der Beugung steht einer spiegelbildlichen Vorstellung von Original und Kopie, von Vor- und Abbild gegenüber, in der es um Fragen der Repräsentationalität geht. Stattdessen verweist das Bild der Beugung auf das In-Relation-Treten von Ereignissen ${ }^{2}$, die gemeinsam eine beobachtbare Veränderung bedeuten (vgl. Deuber-Mankowsky/Holzhey 2013a: 3ff.;

Die Begriffe der Diffraktion und Interferenz werden in der wissenschaftlichen Literatur häufig synonym verwendet, da die Überlagerung von Wellen in der Natur und im Labor praktisch immer mit der Beugung von Wellen zusammen auftritt (vgl. Ziessow/Unkel 2016: 9). Eine reine Wellenbeugung wäre so gesehen ein Sonder- oder Idealfall, weshalb die Unterscheidung der Begriffe von manchen Physikern lediglich als ein »historisches Artefakt« (Barad 2013: 41) betrachtet wird.

2 Der in dieser diffraktiven Denkweise verwendete Begriff des Ereignisses schließt mit DeuberMankowsky (2007: 289) an die Whitehead'sche prozessphilosophische Vorstellung an, die sich von einer substanziell metaphysischen Wirklichkeitsvorstellung abgrenzt und die Wirklichkeit stattdessen in Relationen denkt, in der Ereignisse die Relata darstellen. 
Deuber-Mankowsky 2007: 343f.). Unter einem diffraktiven Leitbild können damit unterschiedlichste Phänomene als ein »interferentes Wirksamwerden« (Bath et al. 2013: 17) und Zusammenspiel ko-konstitutiver Beziehungen sichtbar gemacht werden, innerhalb derer das In-Beziehung-Treten niemals völlig abgeschlossen ist (vgl. Haraway 2003: 12). Damit eignet sich eine diffraktive Perspektive besonders, um den steten Aushandlungsprozess der Bedeutungsgenerierung innerhalb des Phänomens der Handy-Todesvideos in den Blick zu nehmen, an dessen Hervorbringung ästhetische und diskursive Variablen in einer ko-konstitutiven Weise beteiligt sind.

In ihren frühen Arbeiten beschreibt Haraway (2004 [1985]: 23) Diskurse als Formalisierungen im Sinn erstarrter Momente der fließenden sozialen Interaktionen, die sie konstitutieren. Diskurse sieht sie vor allem als Instrumente der Durchsetzung von Bedeutungen. Entsprechend spricht sie auch von Diskursfeldern als Bedeutungsfelder, die sich auf sprachliche und andere materielle Formen der Signifikation stützen und auf unterschiedlichste Weise strukturiert sein können - bspw. als Kotextualität im Sinn geteilter, wenngleich machtdifferenzierter und umstrittener Konversationen oder als Netz sich durchkreuzender und oft gegensätzlicher »inquiries and commitments (Haraway 1989: 349). Diskurse sind damit nicht bloß ein Resultat des Sozialen, sondern haben zugleich fundamentale soziale Effekte, als dass sie Modi der Macht sind (vgl. ebd.: 289). Bedeutungen versteht Haraway (ebd.: 111) hierbei als Anwendungen, die Strukturierungen dessen beinhalten, was zu einer bestimmten Zeit an einem bestimmten Ort als Wissen gelten kann. Die Art und Weise wie Bedeutungen konstituiert werden, gehört für sie daher zum Wesen der Politik. Wenn Haraway hier Diskurse vor allem als materiell-soziale Praktiken der Bedeutungsgenerierung und -durchsetzung versteht, greift sie in ihren folgenden Arbeiten auf das Konzept der Figuration zurück, um die materiell-semiotische Dimension der Bedeutungsgenerierung zu adressieren. Semiose begreift sie hierbei als Politik mit anderen Mitteln. Den wesentlichen Impuls für ihre Konzeption der Figuration - und in direkter Verbindung hierzu für die Vorstellung der Figur -, erhält sie von Eric Auerbach. In seiner Studie der mimetischen Praxis in der westlichen Literatur bestimmt Auerbach Figuration in einem tropischen Verständnis als einen fluiden und reziproken Signifikationszusammenhang zwischen zwei Ereignissen oder Personen, innerhalb dessen sich diese in ihrer Bedeutung gegenseitig bedingen (vgl. Haraway 1997: 10). Entsprechend setzt Haraway Figurationen begrifflich und konzeptionell als performative Bilder sowohl im Sinn immaterieller Vorstellungen als auch verbaler und visueller Materialisierungen in unterschiedlichen medialen Formaten ein, ${ }^{3}$ um die nicht-nachbildende, verschieben-

3 Haraways Konzept der Figuration ist damit anschlussfähig an den Bildbegriff Beltings, der gleichermaßen die materielle und immaterielle Ebene von Bildern adressiert (vgl. hierzu auch Kapitel 1.2). 
de Qualität aller materiell-semiotischen Prozesse sichtbar zu machen. Verwandt mit ihrer frühen, diskursinformierten Betrachtungsweise, behandelt sie Figurationen als verdichtete Karten umstrittener Welten von Bedeutungen, die gelesen und geschrieben werden können und von Figuren getragen werden: »We inhabit and are inhabited by such figures that map universes of knowledge, practice and power.« (ebd.: 11) Ihr tropischer Charakter macht Figuren dabei zugleich wörtlich und metaphorisch, jedoch niemals selbstidentisch, da sie stets eine Form der Verschiebung beinhalten, die Identifikationen und Gewissheiten stören. Als materiellsemiotische Knoten versammeln und verkörpern Figuren geteilte Bedeutungen in Geschichten, die die Leser-, Hörer- und Zuschauerschaft zur selben Zeit bevölkern und diese in den Geschichten verwurzeln. Figuren nehmen also Form innerhalb eines gemeinsam materialisierten narrativen Feldes an, innerhalb dessen sie in einer Verwandtschaftsbeziehung der wechselseitigen Verwirklichung - der TransSubstantion - zueinanderstehen. Zugleich sind sie aber auch Träger von Inhalten, Themen und Bedeutungen, die sich gegenseitig gestalten und prägen. (vgl. Haraway 1997: 11 u. 22f., 2004: 1 u. 2008: 4)

In der diffraktiven Perspektive Haraways (1997: 273) geht es daher gerade nicht darum, eine gleichartige Geschichtlichkeit zu (re-)produzieren, sondern eine Heterogenität von Geschichte bzw. heterogene Geschichten zu ermöglichen: Geschichten, in denen es nicht um "mirroring and straight lines of sight « (Schneider 2002: 472) geht. Geschichten, in denen andere Dinge sichtbar werden als das Bild des Gleichen. Geschichten, in denen die Blicklinien nicht reflexiv zurückgeworfen, sondern gebeugt und verschoben werden und dadurch Relationen und Differenzeffekte sichtbar machen, die ansonsten der Betrachtung entzogen bleiben. Es geht ihr um Geschichten, die kritische Sichtweisen in der Verbindung zu Anderen/m zulassen. Diffraktionsmuster zeichnen entsprechend die Historie von Interaktionen, Interferenzen, Verstärkungen und Differenzen nach. (vgl. Haraway 1997: 273) Bedeutungen resultieren so gesehen aus der figurativen Verschiebung und Überlagerung von Ereignissen innerhalb von Narrativen. Sie sind als generative Differenzen zu verstehen, die sich zu einem Diffraktionsmuster verdichten können. Verdichtet $\mathrm{zu}$ einem narrativen Netz von Bedeutungen, Figurationen und Erzählungen sind sie weder wahr noch falsch. Jedoch können sie als materiell-semiotische Praktiken gewichtig oder ungewichtig sein, als dass sie schwere oder leichte Differenzeffekte in der Hervorbringung bestimmter Sichtweisen und Realitäten nach sich ziehen.

Einer realisierten Wirklichkeit steht so betrachtet ein Raum unrealisierter, aber möglicher, wenn auch nicht notwendiger - sprich: kontingenter - Wirklichkeiten gegenüber, die mit Elena Espositos (1998: 269) systemtheoretischen Ansatz als das Virtuelle bezeichnet werden können, das zum Realen in einem wechselseitigem Be- 
dingungsverhältnis steht. ${ }^{4}$ Esposito bestimmt dieses Verhältnis als eines von verwirklicht und nicht-verwirklicht, aber potenziell verwirklichbar. In diesem Sinn stehe das Virtuelle nicht in Opposition zum Realen, sondern bezeichne »ein oder mehrere Möglichkeitsbereiche neben dem Realen, die es begleiten und zur Unterscheidung zwischen wahr und falsch querstehen (also ihr gegenüber gleichgültig sind)« (ebd.: 270). Zwischen dem Realen und dem Virtuellen verlaufen Espositos Annahme entsprechend keine festinstallierten Grenzen. Vielmehr sind sie als veränderliche und durchlässige Felder vorstellbar, die sich gegenseitig bedingen und in denen die Frage der Aktualisierung von Wirklichkeit eine Frage der relationalen Wahrnehmung und Betrachtung ist (vgl. ebd: 287f.). Die wechselseitige Bedingtheit zwischen Realem und Imaginärem manifestiert sich - wie Haraway (1997: 76ff.) u.a. am Beispiel der >onkomouse und der mit ihr verbundenen >Stalking-Cancer $<-$ Kampagne des US-amerikanischen Konzerns DuPont zeigt - in mitunter monströsen Figuren, die Gestalt in unterschiedlichen medialen Materialisierungen und Artikulationen sowie hochentwickelten Hightech-Produkten annehmen. ${ }^{5}$ Das Imaginäre und das Reale versinnbildlichen hier einander in konkreten Fakten. Die Figuren sind zugleich Kreaturen vorgestellter Möglichkeiten und harter, alltäglicher Realität (vgl. ebd.: 2; 2008: 4). So lassen sie sich mit Esposito als Bewohnende eines virtuellen Möglichkeitsraums verstehen. In diesen Zusammenhang lässt sich auch Haraways Aussage: »the boundary between science fiction and social reality is an optical illusion« (2004 [1985]: 8)« einreihen, womit sie die Trennung zwischen Fakt und Fiktion, zwischen Realem und Imaginärem, zwischen Wahr und Falsch als ein

Ein Umstand, den bspw. Jennifer Eickelmann (2017) in ihrer diffraktiven Analyse der mediatisierten Empörungswellen in den Fällen der Amanda Todd und der Anita Sarkeesian deutlich aufzeigt, wenn sie sichtbar macht, wie virtuelle Praktiken der Hassrede durchaus reale Effekte nach sich ziehen.

5 Haraway (1992) setzt den Begriff des Monsters ein, um die Potentiale (i.S.v. Versprechen, Hoffnungen, aber auch Verheißungen) aufzurufen, die den aus technowissenschaftlichen, biopolitischen und materiell-semiotischen Praktiken resultierenden Cyborgfiguren zugrundeliegen, die unsere sogenannte postmoderne Welt bevölkern. Als Monster leben sie am Rand der Cesellschaft von wo aus es im Abseits des Mainstreams möglich wird neue Sichtweisen und Theorien zu entwickeln. Das Monster lehrt so nicht nur die Norm, wie Deuber-Mankowsky (2013b: 117) mit Verweis auf Canguilhem festhält, sondern auch welche Möglichkeiten die Abweichung von der Norm bereithält, wenn der der Monstrosität innewohnenden Kontingenz Beachtung geschenkt wird. Der Begriff der Artikulation wird hierbei im Sinn Haraways (1992) in Abgrenzung zu dem der Repräsentation verwendet, um sowohl die Vorläufigkeit und Unabgeschlossenheit von Grenzen, auf denen die artikulatorischen Praktiken basieren, zu betonen, als auch um sich von der Besitzergreifung, die repräsentationalen Praktiken innewohnt, zu distanzieren. In der vorliegenden Arbeit wird der Begriff der Artikulation in diesem Sinn gemeinsam mit dem der Materialisierung eingesetzt, um neben textlichen, bildlichen oder audiovisuellen Materialisierungen von Ereignissen auch deren Materialisierungen in mündlichen Konversationen einzuschließen. 
»artifizielles kulturtechnisches Artefakt (Deuber-Mankowsky 2007: 286) illusionären Charakters ausweist. Die kulturtechnischen Verfahren der Hervorbringung von und der Grenzziehung zwischen Fakt und Fiktion, zwischen Realem und Imaginärem, zwischen Wahr und Falsch sind dabei stets als mit politischen Parametern und Ansprüchen verknüpft zu verstehen. So schreibt Haraway den Primatologen Clarence Ray Altmann und Stuart Carpenter auch dieselbe Grundannahme zu wie Science-Fiction-Schriftstellern: »that knowledge is fundamentally political; i.e., dialectically constituted by and constitutive of social possibility.« (Haraway 1989: 111)

Haraway macht dieses wechselseitige Bedingungsverhältnis und die Koexistenz von Imaginärem und Realem in der Science-Fiction namens Wissenschaft durch eine diffraktive Betrachtungs- und Beschreibungsweise sichtbar, in der sie die heterogenen narrativen Verläufe und Bedeutungsgenerierungen gegeneinander beugt und so den Blick auf die Differenzeffekte verschiebt, die in der Interferenz menschlicher und nicht-menschlicher Existenzen erst entstehen (vgl. Haraway 1997: 273; Deuber-Mankowsky/Holzhey 2013a: 25). Was Haraway daher mit ihren Arbeiten vorschlägt und -lebt ist eine andere Art des wissenschaftlichen Schreibens und Sprechens über die Welt, in der sich nicht dem Leitbild der Reflektion und der Reproduktion des Immergleichen verschrieben wird, sondern es um die Hervorbringung und Nachverfolgung folgenreicher Differenzen geht: »Diffraction is a narrative, graphic, psychological, spiritual, and political technology for making consequential meanings. " (Haraway 1997: 273) In diesem Sinn lassen sich ihre Arbeiten auch nicht in die wissenschaftliche Praxis der Produktion eines bestimmten Wissens oder einer bestimmten Wahrheit einreihen. Vielmehr handelt es sich um ästhetische Texte, die einer solchen Praxis widerständig gegenüberstehen und sich ihr vehement verweigern. Wie Deuber-Mankowsky und Christoph Holzhey betonen, spricht sie sich damit etwa »nicht für weniger Wissenschaft, sondern für ein komplexeres Verständnis und eine komplexere Praxis des wissenschaftlichen Wissens« (2013a: 17) aus.

Ebenso wenig wie es dabei Haraways Ansinnen ist, wird im Rahmen dieser Arbeit kein onto-epistemologisches Projekt verfolgt wie es der Anspruch anderer Arbeiten ist (vgl. Deuber-Mankowsky 2011: 89). Prominent ist hier Karen Barad (2007, 2010 u. 2013) zu nennen, die sich Haraways diffraktiver Metaphorik bedient. Diese bettet Barad allerdings in ein quantenphysikalisches Weltbild ein, das ein bestimmtes Wissen und bestimmte Wahrheiten voraussetzt und (re-)produziert. Ihr Anliegen ist es eine neue Ontologie, Epistemologie und - in letzter Instanz - Ethik der Materie bzw. Materialität zu schreiben. Unter diesen Vorzeichen gerinnt eine diffraktive Perspektive jedoch zu einer weiteren wissens(re)produzierenden Technologie, die gerade der Sichtbarwerdung derselben zuwiderläuft. Beispielhaft steht hierfür insbesondere Barads diffraktive Lesart des Quantenradierer-Experiments Quantum Entanglements and Hauntological Relations of Inheritance: Dis/continuities, SpaceTime Enfoldings, and Justice-to-Come (2010). Hier schreibt sie das Quantenradierer- 
Experiment in Verbindung mit Derridas hauntologischem Konzept in eine kammerspielartige Dramaturgie ein, um die Narrative zu stören, die den Geschichten des wissenschaftlichen Fortschritts zugrundeliegen. Sie möchte den Lesenden damit eine gefühlte Wahrnehmung der zugleich verbundenen und zerrissenen, kontinuierlichen und diskontinuierlichen, orientierten und desorientierten Erfahrung der Welt von Elektronen vermitteln, die das Wesen der Quanten-Verschränkung ist. Die dramaturgische Schreibtechnologie selbst wird hier zu einem katalytischen Moment, die ebendiese Wahrnehmung nicht nur hervorbringt, sondern als einzig mögliche voraussetzt:

»The reader should feel free to jump from any scene to another (is there any other way to proceed?) and still have a sense of connectivity through the traces of variously entangled threads and of the (re)workings of mutual constitution and unending iterative reconfigurings (of sections, reader, writer, ideas, ... .). My hope is that what comes across in this dis/jointed movement is a felt sense of différance, of intra-activity, of agential separability - differentiatings that cut together/apart - that is the hauntological nature of quantum entanglements.« (2010: 245)

In Abgrenzung hierzu wird in dieser Arbeit eine Reperspektivierung des wissenschaftlichen Blicks verfolgt, um andere Sichtweisen und Realitäten zu ermöglichen und heterogene Geschichten über das Phänomen der Handy-Todesvideos schreiben zu können. Eine solche diffraktive Perspektivierung setzt dabei eine Situierung der Betrachtung wie auch der Betrachtenden selbst voraus.

\subsection{Die Kontingenz und Situiertheit des Wissens in einer diffraktiven Perspektive}

Die Kontingenz und Situiertheit des Wissens bzw. wissenschaftlicher Tatsachen sind wesentliche Aspekte in Haraways diffraktivem Zugang zur figurativ-narrativen Praxis der Wissens- und Bedeutungsgenerierung. In dieser Praxis ist Wissen über die Welt nicht nur historisch kontingent, sondern eine umkämpfte - mitunter militarisierte - Zone (vgl. Haraway 1989: 111). Haraway verfolgt allerdings keine Fortschreibung dieser Kriegsgeschichte, sondern möchte mit Hilfe des Ansatzes des situierten Wissens den Blick dafür schärfen, dass wir als verkörperte Tatsachen auch hätten anders sein können und noch sein könnten. ${ }^{6}$ Mit Verweis auf Susan

6 In ihrer aktuellen Arbeit Staying with the Trouble (2016) macht es sich Haraway dabei zur Aufgabe eine andere Form der geochronologischen Epoche zu entwerfen: nicht Anthropozän als das Zeitalter des Menschen; nicht Kapitalozän als das Zeitalter der kapitalistischen Produktionsweise, sondern >Chthuluzän ‘ als das Zeitalter der erdverbundenen mythischen Figuren. In dieser wird Erdgeschichte nicht im Sinn der »Man-making tale on a quest to kill and bring back the terrible bounty« (ebd.: 39) und des ewigen Krieges einer heldenhaften Autorität 
Leigh Star hebt Haraway daher hervor, dass in einer Welt, in der es bei Macht darum geht, wessen Metaphern Welten zusammenbringen, die Fragen »where to begin and where to be based « (Haraway 1997: 39) fundamental sind. Denn sobald wir den Chronotopos, der Geschichten auf die eine oder andere bestimmte Weise erzählt, selbst bevölkern, wirkt alles innerhalb dieser Geschichten ausgesprochen real und möglich, vielleicht sogar unausweichlich (vgl. ebd.: 44). Entsprechend sind alle Akteure - menschliche wie nicht-menschliche - innerhalb dieser Wissens- und Bedeutungspraktiken als situiert in »dense worldly webs« (Haraway 2004: 4) vorzustellen.

Wesentlich in einer diffraktiven Perspektive ist daher das Moment der Situiertheit ernst $\mathrm{zu}$ nehmen und sichtbar zu machen, da jedwede Betrachtung und Beschreibung von innen heraus geschieht. D.h. Betrachtende wie auch Betrachtete konstituieren sich wechselseitig innerhalb der Betrachtung und sind selbst Konstituierende der Betrachtung. Eine Situierung des Wissens setzt damit nicht nur voraus, dass die Wissensobjekte als bedeutungsgenerierende Agenten anstatt als bloße Bildfläche oder Ressource der wissensproduzierenden Subjekte verstanden werden. Ebenso müssen die Praktiken, in denen die Wissensobjekte und -subjekte verstrickt sind, als konstituierend für die eigene Position und Interpretierweise begriffen und offengelegt werden, da erst aus diesen Praktiken heraus etwas sichtoder unsichtbar wird. Denn, wie es Haraway ausdrückt: »Accounts of a >reak world do not [...] depend on a logic of >discovery $<$ but on a power-charged social relation of >conversation<. The world neither speaks itself nor disappears in favor of a master decoder.« (Haraway 1988: 592f.)

»I am a marked woman« (Haraway 1997: 2) schreibt Haraway entsprechend gleich zu Beginn in Modest_Witness@Second_Millenium.FemaleMan ${ }^{\odot} \_$Meets_OncoMouse $^{T M}$ und verweist damit auf eben diese Lese- und Schreibtechnologien, die nicht nur sie, sondern uns als legitime Bewohnende der Geschichten ausweisen, die das narrative Netz der christlichen Heilsgeschichten und des technikwissenschaftlichen Fortschritts bilden. Diesem narrativen Netz stellt Haraway ihr eigenes narratives Netz einer diffraktiven Betrachtung gegenüber, in dem ein alternativer "modest witness« (ebd.: 3) als zentrale Figur agiert, der zugleich wissend und unwissend, sorgen- und hoffnungsvoll, misstrauisch und selbst eingebunden innerhalb des Netzes der Geschichten, sagencies« und Apparate ist, die die Technowissenschaft konstituieren. Haraways diffraktive Perspektive setzt sich somit von dem Gestus des distanzierten wissenschaftlichen Blicks eines unsichtbaren, geschichtsfreien >modest witness $<$ b, dessen

namens Wissenschaft geschrieben (vgl. ebd.: 42), sondern als eine Geschichte von »attentive practices of thought, love, rage, and care« (ebd.: 56), die diverse menschliche und nichtmenschliche Akteure in jeder Faser ihres Gewebes einbindet (vgl. ebd.: 55). 
»narratives have a magical power-they lose all trace of their history as stories, as products of partisan projects, as contestable representations, or as constructed documents in their potent capacity to define the facts. The narratives become clear mirrors, fully magical mirrors, without once appealing to the transcendental or the magical.« (ebd.: 24)

Unter diffraktiven Vorzeichen wird dieses >reine< Unterfangen eines "naked way of writing « (ebd.: 26), dem die Autorität transzendentaler Wahrheiten und Fakten durch das Abstreifen der Spuren der Bedeutungsgenerierung unterliegt, durch eine wissenschaftliche Betrachtung und Beschreibung abgelöst, die »finite and dirty« (ebd.: 36) ist. In dieser bleiben die Standorte/-punkte der Betrachtung und Beschreibung sicht- und nachvollziehbar bzw. werden sicht- und nachvollziehbar gemacht. Anstatt also die Situiertheit der eigenen wie der anderen wissens- und bedeutungsgenerierenden Praktiken zu neutralisieren, geht es unter einem diffraktiven Leitbild gerade darum, jedwede Wissens- und Bedeutungspraktik in ihrer je eigenen Situiertheit sichtbar zu machen, den wissenschaftlichen Blick zu verschieben, Bedeutungen gegeneinander zu beugen und übereinander zu legen, und die daraus erwachsenden Differenzeffekte nachzuzeichnen.

Haraway geht es in ihrem Konzept des situierten Wissens darum, die Gesten der Bestimmtheit, Abgeschlossenheit und Universalität, die den Wissenstechnologien der - wie sie es mit Verweis auf Sharon Traweek benennt - »culture of no culture (ebd.: 23) der modernen Wissenschaft abzulegen und stattdessen eine Wissenschaftspraxis partieller und lokalisierbarer Wissensansprüche zu verfolgen (vgl. hierzu auch Schneider 2002: 471). Hierbei orientiert sie sich an Sarah Hardings Konzept der >strong objectivity<, das darauf insistiert, dass sowohl die Objekte als auch die Subjekte der Wissenspraktiken in diesen verortet sein müssen: »Nothing comes without its world, so trying to know those worlds is crucial« ( $\mathrm{Ha}$ raway 1997: 37). Betrachtende können sich nicht abseits der Wissensgenerierung positionieren. Sie können nicht unbeteiligt oder unverbunden gegenüber den bedeutungsgenerierenden Technologien sein. Sich als Betrachtende innerhalb dieser Praktiken zu situieren heißt somit auch verantwortlich und verpflichtet gegenüber der eigenen Betrachtung und Positionierung sowie stets offen gegenüber der (wissenschaftlichen) Auseinandersetzung und Kritik zu sein, anstatt sich ihr in einem relativierenden Objektivierungsgestus zu entziehen.

Haraway grenzt sich damit von einer Wissenschaftspraxis ab, die zwar alle Wissensansprüche als relativ und kontingent sowie alle Wissensobjekte als untrennbar verbunden mit den Praktiken der Wissens- und Bedeutungsgenerierung begreift, dabei jedoch die Wissenssubjekte selbst außerhalb dieser Wissensprakti- 
ken platziert. ${ }^{7}$ Die Wissenssubjekte sind in gleicher Weise wie die Wissensobjekte als Resultate von sich verändernden Grenzziehungen $\mathrm{zu}$ begreifen, d.h. nicht als vorgegebene Entitäten, sondern als historisch kontingent (vgl. Haraway 1988: 595; Deuber-Mankowsky/Holzhey 2013a: 9). Haraways situierter Ansatz handelt somit von einer Kontingenz des Wissens, bei der es um ein verortetes und lokalisierbares Wissen geht; bei der es gerade darum geht, mögliche andere Welten »der einen realen Lebenswelt « (Luhmann 1991 [1984]: 152) hervorzubringen. >Zu wissen` wird so zu einem risikoreichen Projekt: Es geschieht an einem bestimmten Ort, zu einer bestimmten Zeit, von einem bestimmten Körper aus (vgl. Schneider 2002: 469ff.).

\subsection{Entwurf einer diffraktiven Betrachtung und Beschreibung des Phänomens der Handy-Todesvideos}

Das Phänomen der Handy-Todesvideos aus einer diffraktiven Perspektive zu betrachten und zu beschreiben verlangt in der methodischen Umsetzung von je spezifischen Ereignissen - wie einem konkreten Handyvideo, einer konkreten Kunstperformance oder einem konkreten Dokumentarfilm - auszugehen und das in der Betrachtung jeweils erwachsende Beugungsmuster an Geschichten, Figurationen und Bedeutungen in einer medialen Online- und Offline-Umgebung nachzuzeichnen. Diese Geschichten, Figurationen und Bedeutungen verbinden dabei die betrachteten Ereignisse mit anderen Ereignissen. Diese wiederum schreiben die Geschichten fort, verschieben sie oder entwerfen alternative Geschichten, die sich über transnarrative Figurationen und Bedeutungen zu einem narrativen Netz verbinden, aus dem heraus das Phänomen der Handy-Todesvideos sichtbar wird. In der diffraktiven Betrachtung und Beschreibung fügen sich auf diese Weise heterogene, mitunter divergente, Geschichten zu einem eng verwobenen narrativen Netz zusammen, das von Figurationen und Bedeutungen zusammengehalten wird.

Bedeutungen sind dabei im Sinn von Anwendungen $\mathrm{zu}$ verstehen, die generative Differenzen erzeugen: als Unterscheidungen, die zugleich Verbindungen in den Diffraktionsmustern figurativer Geschichten produzieren. Karen Barads Model des Agentiellen Realismus ${ }^{8}$ stellt hierbei die hilfreiche Vorstellung der agenti-

Cegenstand ihrer anhaltenden Kritik ist hierbei der Ansatz von Bruno Latour, dessen AkteurNetzwerk-Theorie sie gerade nicht als Durchbrechung, sondern als Fortschreibung des Narrativs einer Kriegsgeschichte sieht, die Helden und Feinde voraussetzt und hervorbringt (vgl. ebd.: 33ff.; 2016: 42f.).

8 Das von der Quantenphysik ebenso wie von feministischen und poststrukturalistischen Theorien und Ansätzen der >queer theory i informierte Model des Agentiellen Realismus geht von einer grundsätzlichen (und keineswegs unproblematischen) Verschränkung jeglichen Daseins aus. Materie ist hier keine leblose Cegebenheit, die vor der Interaktion ist, sondern ein iteratives, intra-aktives Werden. Weder Individuen noch Materie gehen Realität oder Be- 
ellen Schnitte bereit, mit dem sich das gleichermaßen verbindende und trennende Moment der differentiellen Bedeutungsgenerierung betonen lässt: ein »cutting together-apart, where cuts do violence but also open up and rework the agential conditions of possibility« (vgl. Dolphijn/van der Tuin 2012: 52). Wenn Haraway dabei Figurationen als materiell-semiotische Knoten bestimmt, sollen hier aufgrund der medienkulturwissenschaftlichen Fokussierung der Arbeit und der zentralen Themenfelder des Handyvideos, des Todes und des Syrienkonflikts Figurationen als ästhetisch-diskursive Knoten begriffen werden. Insbesondere lassen sich auf diese Weise die ästhetischen und technologischen Bedingtheiten des Handys bzw. des Handyvideos und der Social-Media-Plattformen ${ }^{9}$, auf denen die Videos Verbreitung finden, ebenso wie diskursive, medienhistorische und ikonografische Bezüge zentral in die Betrachtung einbeziehen.

Da unter einem diffraktiven Zugang weder das Phänomen der HandyTodesvideos selbst noch die es konstituierenden Ereignisse festschreibbar, sondern eingebunden in einer fortwährenden Aushandlung ihrer Bedeutung sind, wird die Betrachtung und Beschreibung zu einer prozesshaften Angelegenheit. Sie gleicht einem Schnappschuss, der im Moment seiner Aufnahme schon vergangen, aber nichtsdestotrotz gegenwarts- und zukunftswirksam ist. Denn Diffraktion handelt vor allem, wie Deuber-Mankowsky betont, von der »Nachträglichkeit und [...] Verbindlichkeit von Ereignissen, die immer schon vorbei sind und anderswo stattgefunden haben« (Deuber-Mankowsky 2007: 345), die in ihrer Wirkung aber eine gegenwärtige und zukünftige Bedeutung tragen. Anstatt also das Phänomen der Handy-Todesvideos, das erst aus der Betrachtung und Beschreibung hervorgeht, zum Ausgangspunkt der Betrachtung zu machen, verschiebt sich der analytische Fokus unter einer diffraktiven Perspektive zu den folgenreichen Differenzen der Beugung und Überlagerung spezifischer Ereignisse, die verdichtet in einem Diffraktionsmuster von Geschichten, Figurationen und Bedeutungen sichtbar werden (vgl. Haraway 1992 u. 1997: 273). Es geht in der Betrachtung und Beschreibung also um die Differenzeffekte von Ereignissen, die dagewesen sind, aber aufgrund ihrer

deutung voraus. Realität stellt sich vielmehr als eine soziomaterielle Praxis der anhaltenden Differenzierung der Welt dar, an der diskursive und materielle, menschliche und nichtmenschliche, natürliche und kulturelle Faktoren reziprok beteiligt sind. (vgl. Barad 2007: 26; Barad/Kleinman 2012: 80)

9 Wichtige Aspekte sind hier bspw. Such- und Vorschlagsalgorithmen, die eine spezifische Serialität der Handy-Todesvideos innerhalb des >media flows ‘ auf YouTube durch die verschlagwortete Verlinkung von Videos hervorbringen. Ebenso sind hier zusätzliche Anwendungsfunktionen hervorzuheben, die das bloße Uploaden und Abrufen von Videos ergänzen (wie z.B. Kommentar-, ssharing «-, sfriending - und Like-Funktionen) und die technologischen Dimensionen der Plattform mit sozioökonomischen und -kulturellen Dimensionen verknüpfen. (vgl. van Dijck 2013: 11ff.; Snickars/Vonderau 2009: 15) 
Flüchtigkeit und Instabilität nur in ihrer differenzbedeutenden Nachträglichkeit als ein zusammenwirkendes Phänomen sichtbar werden. ${ }^{10}$

Spezifische Ereignisse wie ein Handyvideo, einen Dokumentarfilm oder eine Kunstperformanz unter dem übergreifenden Phänomen der Handy-Todesvideos zusammenzufassen und als dieses in seiner Bedeutungsgenerierung zu beschreiben, erscheint damit als eine nachträgliche Pragmatik, die das Ergebnis einer kontingenten und situierten Betrachtung ist. Ändern sich eine, oder mehrere, Variablen können in der Betrachtung und Beschreibung derselben Ereignisse, andere Differenzeffekte als signifikant hervortreten. Diese wiederum verweisen auf wieder andere Ereignisse, machen andere Bedeutungen sichtbar und bringen das Phänomen anders hervor. ${ }^{11}$ Denn es ist ein Sichtbarwerden, das von Wissenspraktiken und -technologien bedingt wird, in denen Betrachtete und Betrachtende gleichermaßen eingebunden und an der Hervorbringung und Aktualisierung von Sichtweisen und Realitäten beteiligt sind. Damit sind weder die in der Betrachtung und Beschreibung erwachsenden Diffraktionsmuster an Geschichten, Figurationen und Bedeutungen verallgemeinerbar, noch diffraktives Betrachten oder Beschreiben an sich standardisierbar. Jedoch lassen sich unter Anwendung einer diffraktiven Perspektive - wie es sich in Anlehnung an Bath et al. (2013: 21) formulieren lässt die Politiken der Bedeutung sicht- und analysierbar machen. Insgesamt können mit einem solchen diffraktiven Vorgehen nicht nur die Bedeutungsgenerierungen der betrachteten konkreten Ereignisse nachverfolgt werden und mit spezifischen ästhetischen Qualitäten und diskursiven Relationen in Zusammenhang gebracht werden. Auch können Aussagen über die Politiken der Bedeutung innerhalb des Phänomens der Handy-Todesvideos in Bezug auf den Tod, das Handyvideo und den Syrienkonflikt getroffen werden. Insbesondere die Verstrickung des Phänomens mit einer umfassenderen Ästhetisierung und Politisierung des Todes lässt sich damit betrachten und beschreiben.

10 Besonders prägnant zeigt sich dieser Umstand an der sich ständig verändernden Verfügbarkeit von potentiell jedem beliebigen Videomaterial, das auf YouTube hochgeladen wird: In einem Moment noch unter einer bestimmten URL und auf einem bestimmten Kanal auf der Plattform abrufbar, kann schon im nächsten Moment dieselbe URL in die Sackgasse eines enttäuscht blickenden Bildschirm-Emoticons führen. Gleichzeitig kann dasselbe Videomaterial in identischer Weise oder in anderen Versionen, Ausschnitten und Videoeinbettungen auf anderen Kanälen weiterhin abrufbar sein. Der Verfügbarkeitsrahmen von Videomaterial auf YouTube folgt damit einer fließenden und unvorhersehbaren Zu- und Abnahme-Logik. In diesem Zusammenhang ist auch zu beachten, dass Social-Media-Plattformen wie YouTube oder Facebook sich stetig verändernden Algorithmen unterliegen, die auf Basis des Nutzerverhaltens festlegen, welche Videos bzw. Inhalte einem spezifischen Betrachtenden angezeigt bzw. empfohlen werden und damit auch bestimmen, welche Videos überhaupt sichtbar werden (vgl. Gielen/Rosen 2016; Seo/Ebrahim 2016). 
Von welchen konkreten Ereignissen die Betrachtung und Beschreibung aus erfolgt und welche weiteren Ereignisse mit diesen innerhalb der Betrachtung und Beschreibung in Beziehung treten, ist dabei selbst Ausdruck der Situiertheit und Kontingenz des Betrachtungs- und Beschreibungsprozesses. Insofern erscheinen die Betrachtung der Ereignisse und die Beschreibung des aus ihr hervorgehenden Diffraktionsmusters an Geschichten, Figurationen und Bedeutungen zunächst zufälligen Charakters zu sein. Allerdings sind weder die Betrachtung noch die Beschreibung beliebig, da sowohl die Ereignisse als auch die Betrachtenden innerhalb des Betrachtungsprozesses, d.h. innerhalb der wissensgenerierenden Praktiken und Technologien, verortet und lokalisierbar bleiben. Denn, wie Deuber-Mankowsky (2007: 291) betont, Diffraktionen treten in keiner unvermittelten, sondern einer kontingenten ${ }^{12}$ Weise in Erscheinung, die sie an spezifische Standpunkte und partielle Sichtweisen knüpft. Die in Beziehung tretenden Ereignisse können dabei in der Vergangenheit, Gegenwart oder Zukunft liegen; d.h. vor dem, gleichzeitig zu dem oder nach dem Erscheinen des Ereignisses, von dem die diffraktive Betrachtung ausgeht. Voraussetzung für das Betrachten und Beschreiben von Diffraktionen ist nur, dass die erwachsenden destruktiven oder konstruktiven Differenzeffekte der Bedeutungsgenerierung in der jeweiligen Betrachtungsgegenwart wirksam und nachspürbar sind. Das so entstehende narrative Netz an Geschichten, Figurationen und Bedeutungen, das das Phänomen der Handy-Todesvideos hervorbringt, bleibt damit unabgeschlossen und stets offen für neue, andere Relationen, d.h. für alternative Differenzeffekte und Bedeutungsverschiebungen.

Die medienkulturwissenschaftliche Fokussierung der Arbeit und das zentrale Themenfeld der Handy-Todesvideos bedingen dabei, dass als Ereignisse mediale Materialisierungen und Artikulationen (wie YouTube-Videos inkl. ihrer Kommentare, Nachrichtensendungen und -artikel, Kunstperformances und -installationen, Dokumentar- und Experimentalfilme) in den Blick kommen, die sowohl auf das Handyvideo als mediale Erscheinungsform als auch auf das Handy als mediale Apparatur des Aufnehmens, Bearbeitens und Verbreitens von Bildmaterial verweisen. Videos in >rohen $<$ Versionen, die mit dem Handy aufgenommen und in unbearbeiteter Form online gestellt wurden, fließen ebenso in die Betrachtung ein wie filmische, künstlerische und videobasierte Auseinandersetzungen, die mit dem Handy

12 Nach Luhmann (1991 [1984]) handelt der Kontingenzbegriff von der »Ausschließung von Notwendigkeit und Unmöglichkeit. Kontingent ist etwas, was weder notwendig noch unmöglich ist. Was also so, wie es ist (war, sein wird) sein kann, aber auch anders möglich ist. Der Begriff bezeichnet mithin Gegebenes (Erfahrenes, Erwartetes, Gedachtes, Phantasiertes) im Hinblick auf mögliches Anderssein. Er bezeichnet Gegenstände im Horizont möglicher Abwandlungen. Er setzt die gegebene Welt voraus; bezeichnet also nicht das Mögliche überhaupt, sondern das, was von der Realität aus gesehen anders möglich ist. [...] Die Realität dieser Welt ist also im Kontingenzbegriff als erste und unauswechselbare Bedingung des Möglichseins vorausgesetzt.«(ebd.: 152) 
aufgenommenes Material beinhalten, in Reaktion auf ein solches produziert wurden und/oder in ihren ästhetischen und diskursiven Effekten figurativ mit einem solchen verstrickt sind.

Mit dem Themenfeld des Todes rücken daneben wissenschaftliche Aussagen zum Tod als soziokultureller Gegenstand wie auch als Gegenstand der Kunst und medialen Auseinandersetzung in den Blick. Aufgrund der gleichzeitigen Fokussierung auf den Syrienkonflikt werden zudem Artikulationen in Form von Aussagen von direkt oder indirekt Betroffenen und Beteiligten des Syrienkonflikts berücksichtigt, mit denen Gespräche geführt wurden. Konkret handelt es sich hierbei um Gespräche mit vier Syrern im Alter zwischen 25 und 45 Jahren, die im Frühjahr 2013 stattgefunden haben. ${ }^{13}$ Im selben Zeitraum wurden auch Gespräche mit dem libanesischen Künstler Rabih Mroué und der deutschen Filmemacherin Birgit Hein in Hinblick auf ihre Werke mit Bezug zum Syrienkonflikt geführt, die ebenfalls Teil der diffraktiven Betrachtung und Beschreibung sind. Hierbei geht es unter dem Leitbild der Diffraktion nicht darum, subjektive Bedeutungen oder Sichtweisen bzw. Wahrnehmungen bestimmter Personen oder Gruppen von einem Ereignis zu zentrieren, sondern um das Nachvollziehen der Hervorbringung und Veränderung von Wahrnehmungen und Bedeutungen, die aus dem In-Beziehung-treten der betrachteten Ereignisse, d.h. der unterschiedlichen medialen Materialisierungen und Artikulationen, entstehen und in der diffraktiven Betrachtung und Beschreibung nachträglich das Phänomen der Handy-Todesvideos hervorbringen.

Unter dem diffraktiven Leitbild wird es mit dem hier formulierten methodischen Ansatz möglich verschiedenste, zuweilen auch divergente, Materialisierungen und Artikulationen in einer Betrachtung und Beschreibung zu vereinen und miteinander in Beziehung zu setzen. Ebenso kann der Fokus innerhalb des Betrachtungsprozesses verschoben und ohne Kohärenzverlust variiert werden, da nicht von einem festgeschriebenen Moment ausgegangen wird, sondern von der Diffraktion von Ereignissen. So können die Differenzeffekte der Bedeutungsgenerierung als ein »relationale[s] Gefüge[...]« (Deuber-Mankowsky/Holzhey 2013a: 7) betrachtet werden, indem mal mehr auf ein spezifisches Medium und seine ästhetischen und technologischen Bedingtheiten oder mal mehr auf die diskursiven Formationen eines bestimmten Ereignisses verwiesen wird. In einem solchen Vorgehen sind dann mögliche Gegensätze nicht als oppositionelle Dualismen zu begreifen, bei denen sich der einen oder anderen Seite zuzuwenden ist, sondern im

Um die Identität der syrischen Cesprächspartner zu schützen, werden in dieser Arbeit keine weiteren Details genannt, die Rückschlüsse auf Person oder Aufenthaltsort zulassen. Soweit es für das Verständnis und die Einordnung der Aussagen der Gesprächspartner notwendig ist, erfolgt eine Kontextualisierung im direkten Zusammenhang der jeweiligen Textpassagen.Weitere Details zur Gesprächsführung und den Gesprächen selbst folgen im nächsten Kapitelabschnitt. 
Sinn eines, wie es Schneider beschreibt, »both, and «(Schneider 2002: 474): Als eine Geschichte, die nicht nach einer privilegierten Berechtigung unserer Beachtung und Betrachtung verlangt. Als eine Geschichte, der nicht die Bürde auferlegt wird, alle rivalisierenden Geschichten bezwingen zu müssen, um als einzig wahre gelten zu können (vgl. ebd.).

Wie es dabei auch Jennifer Eickelmann in ihrer gender- und medienkulturwissenschaftlich ausgerichteten Arbeit »Hate Speech « und Verletzbarkeit im digitalen Zeitalter (2017) für die diffraktive Betrachtung des Phänomens der mediatisierten Missachtung in den Fällen der Anita Sarkeesian und Amanda Todd darstellt, geht es vor dem Hintergrund der leitenden Fragestellungen dieser Arbeit vor allem darum, Geschichten des Todes, des Handyvideos und des Syrienkonflikts im Phänomen der Handy-Todesvideos zu schreiben, die von deren vermeintlichen Bedeutung abweichen. Es geht um »eine perspektivische Verschiebung, so dass Phänomene in anderem Licht erscheinen können« (ebd.: 71). Statt eines abbildenden Schreibens, geht es also um ein >verschiebendes Schreiben, das andere Bedeutungen des Syrienkonflikts, des Todes und des Handyvideos möglich und sichtbar macht. Entsprechend der situierten - d.h. verantwortungsvollen, lokalisierbaren, partialen und kritisierbaren - Positionierung bzw. Verortung als Betrachtende innerhalb des Betrachtungsprozesses ist in der diffraktiven Beschreibung dabei eine Darstellungsweise $\mathrm{zu}$ wählen, die das hierin angelegte Moment eines »always-open engagement of contestation and critique (Schneider 2002: 471) in den Praktiken und Technologien der Wissensgenerierung zu erfassen vermag. Anstelle eines wissenschaftlich distanzierten Schreibstils, der die Schreibenden aus der Beschreibung ausschließt bzw. sich ihnen entzieht und ihre Lokalisierung unmöglich macht, ist bei Haraway >we das operative und kritische Pronomen diffraktiven Schreibens - so unsicher, angreifbar und risikobehaftet es auch sein mag (vgl. Schneider 2002: 473). Im Sinn dieses verantwortungsvollen Eingebunden- und Verbundenseins - eines >being engaged in $<-$ wird in dieser Arbeit ein eben solches diffraktives Schreiben verfolgt, in dem Wir für eine kollektive Opfer-, Täter- und Zeugenschaft des Lebens und Sterbens im Syrienkonflikt steht ebenso wie für eine Gemeinschaft an betrachtenden Mit-Todesleidenden und Mit-zu-Verantwortenden im Syrienkonflikt innerhalb des ko-konstitutiven Zusammenspiels diskursiver und ästhetischer wie auch menschlicher und nicht-menschlicher Variablen.

Das hier eingeführte diffraktive Vorgehen eignet sich für die Betrachtung und Beschreibung des Phänomens der Handy-Todesvideos somit besonders, weil es sowohl die Relationalität und Instabilität seiner konstituierenden Ereignisse adressiert als auch die enge Verwobenheit von medienästhetischen und -technologischen sowie diskursiven Bedingtheiten der Bedeutungsgenerierung zu ihrem Kern macht, anstatt diese als Resultat einer bestimmten wissenschaftlichen Lese- und Schreibtechnik künstlich zu separieren. Damit wird der wissenschaftliche Gestus der projektiven Annäherung an ein Phänomen, der immer in einem 
Außen verweilen muss, abgelöst durch einen Gestus der introjektiven Betrachtung. Die diffraktive Perspektive wird zum konstitutiven Teil des Phänomens selbst.

Angesichts der zu Beginn angedeuteten Komplexität an unterschiedlichen Ereignissen und Bedeutungen des Phänomens der Handy-Todesvideos orientiert sich der Aufbau der Arbeit dabei an den Geschichten und Figurationen, die die betrachteten Ereignisse in einem narrativen Netz verbinden: Jedes Kapitel setzt an einem spezifischen Ereignis des Phänomens der Handy-Todesvideos an - d.h. entweder an einer konkreten medialen Materialisierung oder Artikulation - und verfolgt das hiervon ausgehende Diffraktionsmuster nach, das zusammen mit den Diffraktionsmustern der weiteren Kapitel das Phänomen der Handy-Todesvideos als ein figurativ-narratives Netz der Wissens- und Bedeutungsgenerierung oder anders ausgedrückt - der Politiken der Bedeutung sichtbar werden lässt. Die einzelnen Kapitel sind dabei nicht als undurchlässige, festbegrenzte Bereiche vorzustellen, denen einzelne Ereignisse eindeutig zugeordnet werden können. Vielmehr besteht ein reziproker Verweisungszusammenhang zwischen ihnen, in dem die Ereignisse kapitelübergreifend über transnarrative Figurationen und Bedeutungen in Beziehung zueinanderstehen. >Diffraktiv beschreibt in diesem Sinn auch die Struktur der Arbeit. Das Wiederaufgreifen einzelner Wellen und deren Weiterverfolgung über mehrere Kapitel hinweg ist eine notwendige Konsequenz einer diffraktiven Struktur, die nicht als ein festes Gerüst, sondern als ein bewegliches Gefüge konzipiert ist. Auf diese Weise kann eine Momentaufnahme des Phänomens der Handy-Todesvideos gezeichnet werden, in der der Tod, das Handyvideo und der Syrienkonflikt ausgehend von ihrer Relation zueinander gedacht und vor dem Hintergrund der formulierten Leitfragen gedeutet werden. Innerhalb der einzelnen Kapitel werden hierbei Unterfragen aufgegriffen, die sich während des Betrachtungsprozesses entsprechend der jeweils im Vordergrund stehenden Figurationen und Bedeutungen ergeben und den Fokus der Betrachtung hinsichtlich der erwachsenden Differenzeffekte ständig erweitert, angepasst und ausgerichtet haben.

\subsection{Gesprächsführung unter einer diffraktiven Perspektive}

Unter den hier formulierten diffraktiven Vorzeichen wurde für die bereits erwähnten Gespräche eine freie ethnografische Herangehensweise in Anlehnung an den Soziologen und Kulturanthropologen Roland Girtler (2001) gewählt. Haraway selbst hat dabei die Ethnografie in einem weiten Verständnis (nicht verengt auf ein spezifisches Verfahren der Anthropologie) als eine Methode des »being at risk in the face of the practices and discourses into which one inquires (Haraway 1997: 190) beschrieben. Eine ethnografische Grundhaltung ermögliche es, achtsam und verantwortungsvoll gegenüber den eigenen Risiken, Absichten und Hoffnungen und 
denen der anderen zu bleiben, die in den Praktiken der Wissensgenerierung eingebettet sind (vgl. ebd.: 191). Die Einbeziehung von Gesprächen mit direkt oder indirekt Konfliktbetroffenen und mit Kunst- und Filmschaffenden stellt damit eine konsequente Weiterentwicklung eines diffraktiven Ansatzes dar, der die Politiken der Bedeutung innerhalb einer figurativ-narrativen Praxis der Wissens- und Bedeutungsgenerierung als situierte und lokalisierbare Praktiken nachzeichnen möchte. Die Gespräche treten hierbei als partikulare Erzählungen des Erlebens und der Wahrnehmung von Handyvideos und des Todes im Syrienkonflikt wie auch des Syrienkonflikts selbst in Erscheinung. Sie sind als verortende Momente der Politiken der Bedeutung zu begreifen, die die Wissenssubjekte und -objekte innerhalb der Praktiken der Wissens- und Bedeutungsgenerierung positionieren und diese als zugleich individuelles und kollektives Erleben und Wahrnehmen kartierbar machen.

Die Gespräche fanden entsprechend dieser ethnografisch und diffraktiv informierten Herangehensweise mit der Grundhaltung statt, dass beide Gesprächsseiten sich als Fragende und Erzählende einbringen und das Gespräch möglichst offen und flexibel für unterschiedliche Fragen und Erzählanstöße bleibt. Auf diese Weise konnte nicht nur ein unverbindlicher Raum für die mitunter dramatischen Erfahrungen und Wahrnehmungen der Gesprächspartner geschaffen werden. Auch wurde es möglich sich als Forschende in die Gespräche durch die eigene Positionierung im Sinn eines Sich-in-Beziehung-Setzen mit der Welt (vgl. Deuber-Mankowsky 2007: 287) einzubringen. Damit konnte eine Situierung der Forschenden, der Gesprächspartner und der Bedeutungsgenerierung erfolgen und eine partielle und lokalisierbare Perspektive eingenommen werden, die offen gegenüber der »historische[n] Kontingenz aller Wissensansprüche und Wissenssubjekte« (Deuber-Mankowsky/Holzhey 2013a: 9) und »einer kritischen Positionierung des Wissens« (ebd.: 10) ist.

In den Gesprächen wechselten sich narrative Episoden mit Frage-AntwortEpisoden $\mathrm{ab} .{ }^{14}$ Fragen wurden nicht im Vorhinein festgelegt, sondern entwickelten sich aus dem Gespräch und der Situation heraus und gingen auch von den Gesprächspartnern aus. Somit glichen die Gespräche weniger einem Interview als vielmehr einem Dialog, in dem sich beide Gesprächsseiten gleichwertig gegenüberstanden. Gerade in Bezug auf Ereignisse, die tabu- und affektbehaftet sind, wie es bei Handyvideos von Todesmomenten im Syrienkonflikt der Fall ist, zeigte

Dieses Vorgehen lässt sich mit dem episodischen Interview der qualitativen Sozialforschung vergleichen. Bei diesem werden offene Befragungen und Erzählungen miteinander kombiniert, um sowohl das semantische Wissen als auch das episodische Wissen der Cesprächsteilnehmer zu adressieren. Während semantisches Wissen an Begriffe und ihrer Beziehung zueinander geknüpft ist, ist episodisches Wissen an Erinnerungen an Situationen gebunden. (vgl. Flick 2011: 273f.). 
sich ein solches Vorgehen als sinnvoll. Fragen und Erzählungen waren, wie es auch Girtler (ebd.: 152) in seinem ethnografischen Ansatz beschreibt, geschickt miteinander zu verbinden, indem sowohl Forschende als auch Gesprächspartner wechselseitig die Rolle des Fragenden und Erzählenden einnehmen.

Entsprechend der ethnografischen Ausrichtung des Gesprächsansatzes fand nur die erste Kontaktaufnahme zur gegenseitigen Verständigung auf einen Ort und Zeitpunkt für ein persönliches Treffen per E-Mail statt. Mit dem Anspruch eine persönliche und vertrauliche Atmosphäre während der Konversation zu schaffen, wurden die Gespräche nach den Wünschen der Gesprächspartner entweder in deren Wohn- oder Arbeitsräumen oder in einem öffentlichen Lokal ihrer Wahl geführt. Die Gespräche wurden nach ausdrücklichem Einverständnis der Gesprächspartner mit einem digitalen Diktiergerät aufgezeichnet und nach der Transkription einer fortlaufenden Auswertung während des Betrachtungs- und Beschreibungsprozesses unterzogen, indem die Äußerungen kontinuierlich hinsichtlich ihrer figurativ-narrativen Beziehung zu den betrachteten Ereignissen begangen wurden und die daraus erwachsenden Differenzeffekte für die Bedeutungsgenerierung Eingang in den Betrachtungs- und Beschreibungsprozess fanden.

Damit fügt sich diese Herangehensweise in das Leitbild der Diffraktion ein. Sie folgt nicht einer traditionellen wissenschaftlichen Operationalisierung oder einer metaphysisch basierten Wissensobjektivierung, wie es sich in klassischen FrageAntwort-Schemata wiederfindet in denen es um das Testen von vorbestimmten Sichtweisen und Hypothesen geht (vgl. Gitler 2001: 50f.; Baudrillard 2011 [1976]: 113ff.). Vielmehr brachten die Gespräche in ihrem narrativ-dialogischen Moment Differenzeffekte hervor, die im überlagernden Zusammenspiel der Wellenbewegungen, die von den unterschiedlichen medialen Materialisierungen ausgehend den Blick auf Bedeutungen des Todes, des Handyvideos und des Syrienkonflikts gebeugt haben, die andernfalls außerhalb der Betrachtung standen. Diese Differenzeffekte wirkten bezogen auf bestimmte Bedeutungen des Todes, des Handyvideos und des Syrienkonflikts verstärkend oder abschwächend und damit perspektivisch verschiebend. Die Gespräche treten somit einerseits als in Beziehung tretende Ereignisse innerhalb von Diffraktionsmustern in Erscheinung, die destruktive oder konstruktive Differenzeffekte in der Bedeutungsgenerierung nach sich ziehen. ${ }^{15}$

15 Insbesondere sind hier auch die ästhetischen und diskursiven Bedingungen des Gesprächskontexts zentral, in denen die räumlichen und zeitlichen Gegebenheiten ebenso hineinspielen, wie die wissensgenerierenden Techniken der Gesprächsführung. Ein besonders markantes Beispiel liefert hier das anwesende Diktiergerät, das als digitaler Mithörer und Tonschreiber sowohl Einfluss auf die Cesprächssituation als auch auf den Prozess der Bedeutungsgenerierung nahm, da mit ihm eine spezifische Möglichkeit der Übersetzung mündlicher Artikulationen in schriftliche Materialisierungen geleistet wurde. Das Diktiergerät brachte auch Diskurse der Überwachung und des Verhörs in die Gespräche ein, welche insbesondere in Hinblick auf eine häufig konstatierte Internierungspraxis des syrischen Regimes von Rele- 
Damit werden sie ein konstitutiver Teil der verfolgten diffraktiven Perspektive. Andererseits sind sie als ein Effekt derselben diffraktiven Perspektive zu sehen, wenn sie erst aus der Betrachtung und Beschreibung heraus entstehen. Indem die Sichtweisen direkt und indirekt konfliktbetroffener Syrer, eines libanesischen Künstlers und einer deutschen Filmemacherin mit der einer Medienkulturwissenschaftlerin in Dialog treten, spannt sich somit ein komplexes und vieldeutiges narratives Netz interferierender ästhetischer und diskursiver Effekte auf.

Zum Zeitpunkt der Gespräche waren die syrischen Gesprächspartner dabei entweder bereits seit mehreren Jahren in der Bundesrepublik Deutschland ansässig oder lebten erst seit kurzem in der Bundesrepublik (weniger als ein Jahr) auf der Basis von Arbeitsvisa. Der Kontakt zu den syrischen Gesprächspartnern wurde über persönliche Netzwerke und das offizielle Netzwerk des Flüchtlingsrats Nordrhein-Westfalen e.V. hergestellt. Die einzigen Auswahlkriterien waren eine direkte oder indirekte Betroffenheit durch den Syrienkonflikt und irgendeine Form der Erfahrung mit Handyvideos im Syrienkonflikt. ${ }^{16}$ Im Gegensatz dazu erfolgte die Kontaktaufnahme mit Rabih Mroué und Birgit Hein direkt über E-MailAdressen, die auf ihren persönlichen und werkbezogenen Internetauftritten verfügbar waren. Je nach Sprachkenntnissen der Gesprächspartner wurden die Gespräche auf Deutsch oder Englisch geführt. Eine Gesprächsführung nicht in der Muttersprache der syrischen Gesprächspartner zeigte sich als wesentlicher Einflussfaktor der Konversationen, wenn bspw. eine niedrige Sprachkompetenz den Gesprächspartner in seiner Aussagefähigkeit beschränkte und den verstärkten Einsatz von Gesten und Wörterbüchern zur Folge hatte oder Ausdrücke und Wendungen in der jeweiligen Fremdsprache verwechselt wurden. Um die allgemeine Verständlichkeit der Aussagen zu gewährleisten, werden die Aussagen der syrischen

vanz sind (vgl. hierzu bspw. ROC 2015). Entsprechend war das Diktiergerät als Dokumentationsapparat von als delinquent bewertbarer Aussagen mit einem besonderen Risiko und einem eigenen Moment der Vulnerabilität für die syrischen Cesprächspartner verbunden. Auch steht das Diktiergerät mit einer Ceständnispraxis in Relation, die nach Foucault (2008 [1976]: 1071ff.) eine spezifische wahrheitsproduzierende Technik im wissenschaftlichen Diskurs ist und mit einer besonderen Machtwirkung bzw. mit der Etablierung einer besonderen Form der Machtbeziehung zwischen Sprechendem und Zuhörendem einhergeht. Im Einklang hiermit waren auch eine gewisse Erleichterung und Entspannung bei den syrischen Cesprächspartnern zu beobachten als das Diktiergerät am Ende des Cespräches ausgeschaltet wurde.

16 Drei der vier syrischen Cesprächspartner waren dem Konflikt in Syrien direkt ausgesetzt. Der Konflikt wirkte sich stark auf ihr Leben in Syrien aus und zwang sie das Land 2014 oder 2015 zu verlassen. Alle drei gaben an, selbst keine Videos vom Konflikt aufgenommen zu haben jedoch Personen zu kennen, die solche Videos aufgenommen und verbreitet hatten. Der vierte Cesprächspartner hatte Syrien bereits vor Ausbruch des Konflikts für ein Studium in Deutschland verlassen, steht aber seither mit Verwandten und Freunden in Syrien - von denen einige selbst Videos aufnehmen und verbreiten - über soziale Medien in Kontakt. 
Gesprächspartner in dieser Arbeit daher nicht wortwörtlich, sondern in einer vereinfachten Form wiedergegeben, die auch extralinguale Aspekte und interpretative Leistungen in Hinblick auf den Gesamtzusammenhang des Gesprächs beinhalten.

Aufgrund dieser stark zum Tragen kommenden extralingualen und interpretativen Momente und der damit verbundenen eigenen Situiertheit der Gespräche, wird des Weiteren auf eine Veröffentlichung der Transkriptionen verzichtet. Als situierte Ereignisse sind die geführten Gespräche in gleicher Weise wie die HandyTodesvideos einer fortwährenden Aushandlung ihrer Bedeutung ausgesetzt, die stets nur als Effekt einer spezifischen Betrachtung und Beschreibung in Erscheinung tritt. Das eigene >being engaged in und >being at risk als Forschende infolge der aktiven Involviertheit in den Gesprächen mit einer eigenen wissenschaftlichen und soziokulturellen Situierung macht es nahezu unausweichlich, dass andere Betrachtende andere Bedeutungen in den transkribierten Aussagen der Gesprächspartner lesen. Ebenso wenig sind die mitunter verzerrenden Effekte der Übersetzungsleistung eines gesprochenen Dialogs in einen Schrifttext, der die Intonation, Mimik und Gestik sowie die mitschwingenden Emotionen nur unzulänglich zu erfassen vermag, hinsichtlich potentieller Bedeutungsverschiebungen nicht zu vernachlässigen. Ein mit einer Veröffentlichung einhergehendes potentielles Heraustrennen aus dem sich hier entfaltenden figurativ-narrativen Netz und eine mögliche entkontextualisierende und reinterpretierende Wieder- und Weiterverwendung von transkribierten Artikulationen erscheint auch insbesondere in Hinblick auf die Einwilligung der Gesprächspartner in die Gespräche und deren Aufzeichnung ausschließlich für den Rahmen des Promotionsprojekts als nicht vertretbar. Schließlich darf angesichts der politischen Parameter im Syrienkonflikt nicht übersehen werden, dass eine Veröffentlichung der Gesprächstranskriptionen ein unüberschaubares Risiko für die syrischen Gesprächspartner darstellt. Einer möglichen Forderung nach wissenschaftlicher Transparenz ist damit das persönliche Interesse der syrischen Gesprächspartner gegenüberzustellen, die sich mit der Einwilligung in ein offenes Gespräch zu ihren Erfahrungen, Eindrücken und Meinungen zu den Handyvideos, aber auch zum Syrienkonflikt, einem gewissen Risiko der politischen Verfolgbarkeit aussetzen. Dieses Risiko sollte den Rahmen dieses persönlichen Gesprächs möglichst nicht überschreiten. Angesichts der nichtsdestotrotz legitimen Ansprüche an eine wissenschaftliche Transparenz können auf Anfrage und vorbehaltlich der einzuholenden Zustimmung der Gesprächspartner, die Gesprächstranskriptionen in Einzelfällen zur Verfügung gestellt werden.

Nach dieser theoretischen und methodischen Einführung wird sich nun im zweiten Teil der Arbeit den heterogenen Geschichten, Figurationen und Bedeutungen gewidmet, die das figurativ-narrative Netz des Phänomens der HandyTodesvideos bilden. Kapitel drei fokussiert Ereignisse, die Geschichten des Todes, des Handyvideos und des Syrienkonflikts in der 1. Person erzählen, d.h. die die Perspektive der Filmenden und Sterbenden im Syrienkonflikt wiedergeben. Zunächst 
ausgehend von dem im ersten Kapitel vorgestellten netspanner-Video und die hieran anschließende `non-academic Lecture/Performance`von Rabih Mroué, fällt unser beugender Blick auf die Artikulationen aus den Gesprächen mit Syrern ebenso wie auf die ikonische Filmaufnahme von Leonardo Henrichsen aus dem Jahr 1973, die ästhetisch und diskursiv mit dem netspanner-Video verflochten ist. Von dem hier kartierten Diffraktionsmuster, führt uns die Betrachtung sowohl weiter zu anderen >First-person<-Handy-Todesvideos aus dem Syrienkonflikt als auch zu den filmischen Auseinandersetzungen von Birgit Hein sowie von Ossama Mohammed und Wiam Simav Bedirxan. Kapitel vier wiederum nimmt Ereignisse in den Blick, in denen von einer Erzählperspektive der 3. Person gesprochen werden kann, d.h. von der Perspektive der Anderen, die den Tod und das Sterben im Syrienkonflikt filmen und betrachten. Ansetzend an >Third-person<-Handy-Todesvideos aus dem Syrienkonflikt, die über eine religiöse und mythologische Motivgeschichte an die ikonischen Todesbilder des Che Guevara und des Benno Ohnesorg anschließen, werden wir in der diffraktiven Betrachtung erneut zu dem Tod der Neda AghaSoltan getragen. Von hier aus führen uns die figurativen Verflechtungen weiter zu Videoereignissen, die als >leaked und enthüllende Dokumente in Erscheinung treten und mit gegenläufigen Erzählungen aus den Gesprächen mit Syrern interferieren. Auch gelangen wir zu den IS-Enthauptungsvideos westlicher Staatsangehöriger aus dem Jahr 2014 von denen aus sich ein komplexes Diffraktionsmuster mit Bezügen $\mathrm{zu}$ einer jihadistischen Ikonografie und einer selbstexponierenden Praxis des Betrachtens ausbreitet. Gleichzeitig verflechtet sich dieses mit Diffraktionsmustern, die von parodistischen und rechtspopulistischen Videoereignissen ausgehen.

Die Unterscheidung zwischen >First-person<- und >Third-person<-HandyTodesvideos ist dabei nicht bloß das Ergebnis einer analytischen und schreibtechnischen Pragmatik. Vielmehr zeichnet diese den eigenen Blick der wissenschaftlichen Betrachterin nach. Dieser nahm 2012 seinen Anfang mit der künstlerischen Arbeit von Rabih Mroué und setzte damit an Handyvideos an, die den Tod aus der POV-Perspektive zeigen. Von dort aus richtete er sich zunächst auf Handyvideos, die den Tod der Filmenden im Syrienkonflikt oder den soziopolitisch verwandten Konflikten in der MENA-Region zu dieser Zeit festhalten und in unterschiedlichen künstlerischen und filmischen Werken reproduziert und zitiert werden. Ebenso tauchte er in eine Motivgeschichte der Bilder des Todes im Kriegs- und Konfliktkontext ein und wurde eng mit dem aus einer Beobachterperspektive aufgezeichneten Tod der Neda Agha-Soltan verflochten. Mit Erscheinen der ISEnthauptungsvideos im Jahr 2014 erhielt dieser Blick dann eine neue, folgenreiche Stoßrichtung: Mit dieser politisch aufgeladenen Reperspektivierung auf den Tod des Anderen verschob sich der Blick und ließ andere Geschichten, Figurationen und Bedeutungen in den Fokus rücken, die das Phänomen und die sich mit ihm ausbreitenden Wellenmuster veränderten. Die folgenden zwei Kapitel sind in 
diesem Sinn auch als Kartierungen des Wegs zu verstehen, den der Blick einer wissenschaftlichen Betrachterin mit dem Phänomen der Handy-Todesvideos genommen hat, wie dieser mit dem Phänomen verändert und hervorgebracht wurde, und wie dieser wiederum das Phänomen selbst verändert und hervorbracht hat. 
